\title{
HLA-A*02-B*46 Haplotype: an Adverse Prognostic Factor in Han Patients with Nasopharyngeal Carcinoma
}

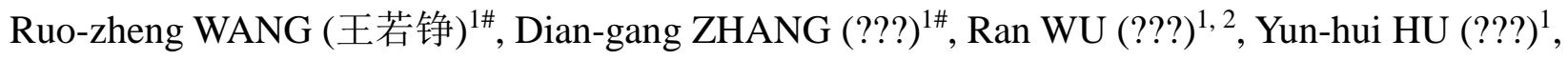

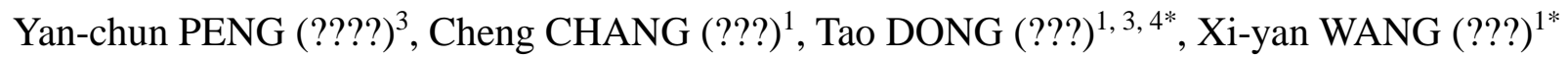

${ }^{1}$ Department of Head and Neck Radiation Oncology, The Affiliated Tumor Hospital of Xinjiang Medical University, Urumqi, Xinjiang 830011, P.R. China

${ }^{2}$ Oncology Department, Bazhou People’s Hospital of Mongolian Autonomous Prefecture, Kuerle, Xinjiang 841000, P.R. China

${ }^{3}$ MRC Human Immunology Unit, Radcliffe Department of Medicine, Oxford University, Oxford, OX3 9DS

${ }^{4}$ Nuffield Department of Medicine, Oxford University, Oxford, OX3 9DU Ruo-zheng WANG, E-mail: ????; Dian-gang ZHANG, E-mail: ????

\#The authors contributed equally to this work.

${ }^{*}$ Corresponding authors, Tao DONG, E-mail: tao.dong@imm.ox.ac.uk; Xi-yan WANG, E-mail: wrz8526@163.com

This project was supported by grants from the Chinese International Cooperation Project (No. 2012DFA31560), Key Laboratory Projects of Xinjiang Uygur Autonomous Region (No. 2015KL021), and the Autonomous Region Achievement Promotion Projects (No. 201554142).

\section{Conflicts of Interest Statement}

The authors have no financial conflicts of interest.

Summary: Epidemiological studies have shown that human leukocyte antigen (HLA) allelic polymorphisms are closely correlated to susceptibility to nasopharyngeal carcinoma (NPC), and in a previous study, we showed that HLA-B*46 and HLA-A*02-B*46 haplotypes were strongly associated with NPC susceptibility. In this retrospective study, we investigated the phenotype of the HLA-A and HLA-B alleles and haplotypes and correlated these data to the clinical and pathological parameters of NPC to understand the role of HLA alleles and haplotypes in NPC prognosis. The cohort comprised 117 NPC patients from a Han population in Xinjiang. The local recurrence-free survival (LRFS), distant metastasis-free survival (DMFS), disease-free survival (DFS), and overall survival (OS) were analyzed. The 5-year DMFS of the HLA-A*02-B*46 haplotype carriers and 
non-carriers was $66.4 \%$ and $90.3 \%$, respectively. In addition, age was found to be a prognostic factor for LRFS, DFS, and OS $(P=0.032,0.040$, and 0.013 , respectively). We found that the HLA-A*02-B*46 haplotype might be a prognostic marker in addition to the traditional TNM staging in patients with NPC.

Key words: human leukocyte antigens; phenotype; nasopharyngeal carcinoma; prognosis; metastasis

Nasopharyngeal carcinoma (NPC) is classified as a head and neck malignant cancer originating from the mucosal epithelium of the nasopharynx. The distribution of NPC varies markedly by geography, ethnicity, and familial aggregation. In addition to Epstein-Barr virus (EBV) infection and environmental factors, genetic factors have been recently shown to play a role in the pathogenesis of NPC ${ }^{[1]}$. 下同 The human leukocyte antigen (HLA) system includes gene loci located on the short arm of chromosome 6 at 6p21.3. HLA genes play a vital role in antigen processing and presentation as well as the anti-tumor immune response. ${ }^{2}$ Epidemiological studies have shown that HLA allelic polymorphisms are closely correlated with susceptibility to NPC. ${ }^{3-5}$ In a previous study, we investigated the HLA genotypes of 132 NPC patients and 168 normal controls of Han ethnicity in Xinjiang Province and found that the HLA-B*46 and HLA-A*02-B*46 haplotypes were strongly associated with NPC susceptibility. ${ }^{6}$ In this study, we further investigated the role of HLA alleles and haplotypes in NPC prognosis in a Han population in Xinjiang.

\section{MATERIALS AND METHODS}

\subsection{Study Subjects}

In total, 117 (81 men, and 36 women) Han NPC patients (age range, 11-81; mean age, 48.03 years), who received intensity-modulated radiation therapy at the Department of Head and Neck Radiation Oncology of the Affiliated Tumor Hospital of Xinjiang Medical University between July 2007 and July 2011 were included in this study. ${ }^{6}$ The association of HLA-A/B alleles and haplotypes with the clinical outcome of NPC was investigated. Patients with distant metastasis and incomplete clinical history were excluded from the study. According to the World Health Organization pathological classifications, 5 patients had type I differentiation (squamous cell carcinoma), 43 had type II differentiation (non-keratinizing carcinoma), and 69 had type III differentiation (undifferentiated carcinoma). The Union for International Cancer Control-American Joint Committee on Cancer 2002 staging system was used to determine the clinical stage. 
Follow-up was scheduled every 3 months in the first 2 years, every 6 months for 3-5 years, and annually thereafter. The last follow-up date was June 30, 2014. Six individuals were lost; thus, the follow-up rate was $94.9 \%$. The clinical characteristics of the patients are listed in table 1.

\subsection{Ethical Considerations}

This study was approved by the institutional ethics committee of the Affiliated Tumor Hospital of the Xinjiang Medical University with approval number G-201103042. Written informed consent was obtained from each participant included in this study.

\subsection{Genomic DNA Extraction}

Venous blood was obtained from the patient before the start of therapy and stored at $-20^{\circ} \mathrm{C}$ before analysis. Genomic DNA was extracted according to standard protocols ${ }^{7}$ by using a Genomic DNA Extraction kit (Bioteke, China) following the manufacturer's protocol. DNA concentration and purity were determined using an ultraviolet spectrophotometer (Thermo, Finland). The A260/280 ratio was $1.8-1.9$ with an adjusted final concentration of $0.3-0.5 \mu \mathrm{g} / \mu \mathrm{L}$. HLA-A and HLA-B genotyping was performed by the Beijing Institute of Genomics (BGI, China) by using high-throughput HLA sequence-based typing.

\subsection{EBV Viral Capsid Antigen IgG Assay}

Plasma (in EDTA) from the NPC patients was separated from the blood and stored at $-20^{\circ} \mathrm{C}$. The EBV viral capsid antigen IgG kit (Demeditec, Germany) was used for detection and quantization of human IgG antibodies against EBV viral capsid antigen in the plasma by following the manufacturer's protocol.

\subsection{Statistical Analysis}

Statistical analysis was performed using SAS 9.3 software. The 5-year overall survival (OS), local recurrence-free survival (LRFS), distant metastasis-free survival (DMFS), and disease-free survival (DFS) rates were evaluated using the Kaplan-Meier method. Univariate analysis was performed using the log-rank test. The Cox proportional hazards model was used to analyze the relationship between the different factors and prognosis for multivariate analysis. 


\section{RESULTS}

\subsection{Survival Rate of NPC Patients}

The mean duration of follow-up was 61 months (7-84 months). The 5-year LRFS, DMFS, DFS, and OS were $84.9 \%, 80.1 \%, 69.2 \%$, and 82.2\%, respectively. Furthermore, 15 and 20 patients developed local recurrence and distant metastasis, respectively; 17 patients died.

\subsection{Association of Clinical Characteristics and NPC Prognosis by Univariate Analysis}

The effects of age, sex, and smoking on NPC prognosis were determined by dividing the study subjects into the corresponding groups: age, <50 and >50 years; men and women; and smoking and non-smoking groups. Our results showed that patients aged $<50$ years had a better survival rate in terms of LRFS, DFS, and OS than those aged $>50$ years $(P=0.011,0.025$, and 0.006, respectively). Furthermore, the impact of clinical characteristics, including World Health Organization pathological type, $\mathrm{N}$ and $\mathrm{T}$ stage, and clinical staging, on NPC patient survival rates was evaluated. Among all clinical factors, N stage was a potential prognostic factor for LRFS, DMFS, DFS, and OS $(P=0.024,0.011,0.005$, and 0.017 , respectively). Furthermore, T stage had a marked impact on prediction of DMFS and DFS ( $P=0.048$ and 0.009 , respectively).

\subsection{Association between HLA Alleles and Haplotypes and NPC Prognosis by Univariate} Analysis

In total, 14 and 25 alleles on HLA-A and HLA-B loci, respectively, were studied, and 123 haploids on HLA-A/B alleles were detected. HLA-A24 and HLA-B46 alleles had an insignificant effect on NPC survival parameters. The A2-B46 haplotype, a known NPC risk factor, was also found to be strongly associated with 5-year DMFS in NPC patients $\left(\chi^{2}=8.362, P=0.003\right)$. Furthermore, 33 patients belonged to HLA-A*02-B*46 (28.2\%); among them, 15 (45.5\%) developed distant metastasis. The 5-year DMFS of the HLA-A*02-B*46 haplotype carriers and non-carriers was $66.4 \%$ and $90.3 \%$, respectively (table 1). The association between different clinical stages such as stages I-II and III-IV and NPC outcomes was also analyzed. The HLA-A*02-B*46 haplotype was found to be markedly correlated with the 5-year DMFS in NPC patients at stage IIIIV $\left(\chi^{2}=6.276, P=0.012\right)$. The 5-year DMFS of the HLA-A*02-B*46 haplotype carriers and non-carriers for clinical stage III-IV NPC patients was $62.5 \%$ and $87.8 \%$, respectively (fig. 1). 


\subsection{Multivariate Analysis}

Multivariate analysis indicated that age was an independent prognostic factor for LRFS, DFS, and $\mathrm{OS}\left(\chi^{2}=4.613,4.231\right.$, and 6.210; $P=0.032,0.040$, and 0.013 , respectively) in the NPC population. $\mathrm{N}$ stage can be used as a potential predictive factor for DMFS, DFS, and OS $\left(\chi^{2}=4.258\right.$, 5.943, and 5.634; $P=0.039,0.014$, and 0.018 , respectively). The HLA-A*02-B*46 haplotype was also strongly associated with DMFS $\left(\chi^{2}=5.027, P=0.024\right)$ in the NPC patients (table 2$)$.

\section{DISCUSSION}

EBV infection is known to be closely associated with the development of NPC by contributing to the pathogenesis of poorly differentiated and undifferentiated pathological types of carcinoma. NPC has unique pathological characteristics, has distinctive sensitivity to radiotherapy, and is highly invasive, metastatic, and malignant. ${ }^{8}$ Some NPC patients in complete remission develop distant metastasis and local recurrence, leading to treatment failure. Hence, identifying new prognostic molecular markers for the treatment and prognosis of NPC patients is important.

The EB virus-specific immune response after infection plays an important role in the anti-tumor process of humans. HLA genes are responsible for the regulation of the major gene-specific immune system in humans and can be divided into HLA class I (A, B, C), class II (DR, DQ, DP), and class III (complement component coding genes). HLA class I genes are located on the surface of nucleated cells in vivo; they recognize and present endogenous antigens and participate in the $\mathrm{CD}^{+}$cytotoxic $\mathrm{T}$ lymphocyte (CTL)-mediated response via the endogenous processing pathway. They exert their biological effects via the cell-mediated immune response and play an essential role in T-cell-mediated anti-tumor immunity. ${ }^{9}$ Despite recent advances in understanding the role of HLA alleles and haplotypes in NPC susceptibility, little is known regarding their prognostic value or association with patient outcome. Yuan et al. observed that HLA-I down-regulation is associated with tumor progression and a poor prognosis in clear cell renal cell carcinoma (CCRCC) patients. ${ }^{10}$ In particular, they showed a significant correlation of HLA-I expression with TNM stage, lymph node metastasis, and Fuhrman grade in CCRCC. Patients with tumors displaying down-regulation of HLA-I showed significantly shorter overall survival $(P=0.021)$. 
Anti-cancer immunotherapy is considered a promising treatment modality for metastatic renal cell carcinoma (mRCC), as up-regulation of erythropoietin (Epo) and its receptor (EpoR) is involved in tumorigenesis in RCC. Minami et al. found that the novel EpoR-peptides EpoR52-60 could be promising candidates for a peptide-based anti-cancer vaccine for mRCC patients carrying HLA-A24. ${ }^{11}$

Tertipis et al studied 425 patients with tongue and tonsil cancer and found that HLA-A*02 non-carriers had a higher DFS rate than HPV-positive patients. ${ }^{12}$ Simons et al. also showed that NPC patients with HLA antigen A2 in the absence of B-Sin 2 (BW46) or BW17 had a better prognosis. ${ }^{13}$ In this study, the HLA-A*02-B*46 haplotype was found to be an independent prognostic factor for DMFS, and HLA-A*02-B*46 haplotype carriers had lower DMFS than non-carriers. However, no associations between HLA-A and HLA-B alleles and disease prognosis were found in the NPC patients in this study. This discrepancy in results may have resulted from insufficient sample size, genetic differences, or other factors related to the frequency distribution of HLA alleles.

Numerous studies have shown that HLA-A*02-B*46 is the most common NPC-associated haplotype and shows significant linkage disequilibrium in NPC populations from southern China, Taiwan, and other Asian countries. 5, 14, 15 Our previous studies have also confirmed that HLA-A*02-B*46 haplotype is correlated with NPC susceptibility in Xinjiang. ${ }^{6}$ Nevertheless, whether or not HLA-A*02-B*46 is associated with disease prognosis remains largely unknown. Linkage disequilibrium theory, antigen presenting theory, and immune escape theory have been suggested to underlie the association of HLA with NPC susceptibility. ${ }^{9,}{ }^{16,17}$ However, whether or not these mechanisms contribute to the impact of HLA alleles and haplotypes on NPC prognosis warrants further investigation. HLA-A*02-B*46-expressing cells have been hypothesized to show weak antigen-presentation capacity and thus evade the cytotoxic T lymphocyte-mediated anti-tumor immune response in the body, leading to the adverse clinical progression in HLA-A*02-B*46-positive NPC individuals.

Consistent with the findings of a previous study by Han et al, our data also showed that age and $\mathrm{N}$ stage were the predominant prognostic factors in Han NPC patients in Xinjiang. ${ }^{18}$ Hence, traditional TNM staging combined with HLA-A*02-B*46 haplotype and other prognostic molecular markers might be helpful to better predict NPC prognosis. Phenotyping of HLA-A and 
HLA-B alleles and haplotypes in NPC patients can allow HLA-A*02-B*46 carriers to be treated with targeted interventions to reduce the rate of distant metastasis; thus, genetic and immunological findings can help develop novel prognosis and treatment strategies for NPC.

With recent advances in microsatellite loci genotyping technology, genetic statistics, and molecular biology, elucidating the association between HLA and NPC prognosis might become possible. Future large-scale prospective studies with multi-center clinical research collaboration need to be performed to further investigate the impact of other HLA alleles, haplotypes, or recessive genes in the HLA region on NPC prognosis. 


\section{REFERENCES}

\section{请参考文献 1-4 对后面的文献进行规范和统一}

1 Tang MZ, Cai YL, Zheng YM, et al. Association between human leukocyte antigen and nasopharyngeal carcinoma. Yi Chuan (Chinese), 2012,34(12):1505-1512

12 Horton R, Wilming L, Rand V, et al. Gene map of the extended human MHC. Nat Rev Genet, 2004,5(12):889-899

23 Chin YM, Mushiroda T, Takahashi A, et al. HLA-A SNPs and amino acid variants are associated with nasopharyngeal carcinoma in Malaysian Chinese. Int $\mathbf{J}$ Cancer, 2015,136(3):678-687

$34 \mathrm{Yu} \mathrm{KJ}$, Gao X, Chen CJ, et al. Association of human leukocyte antigens with nasopharyngeal carcinoma in high-risk multiplex families in Taiwan. Hum Immunol, 2009,70(11):910-914

4 Tang M, Zeng Y, Poisson A, Marti D, Guan L, Zheng Y, et al. Haplotype-dependent HLA susceptibility to nasopharyngeal carcinoma in a Southern Chinese population. Genes Immun 2010;11(4):334-42.

5 Wang R, Hu Y, Yindom LM, Huang L, Wu R, Wang D, et al. Association analysis between HLA-A, -B, -C, -DRB1, and-DQB1 with nasopharyngeal carcinoma among a Han population in Northwestern China. Hum Immunol 2014;75(3):197-202.

6 Lin Z, Guo Z, Xu Y, Zhao X. Identification of a secondary promoter of CASP8 and its related transcription factor PUR $\alpha$. Int J Oncol. 2014;45(1):57-66.

7 Luo RZ, Zhong BL, Zong YS, Ruozheng W, Hu Y, Yindom L-M, et al. Histopathological characteristics of nasopharyngeal carcinoma in high incidence areas. Chin J Cancer $2001 ; 8: 473-5$.

8 Tang M, Lautenberger JA, Gao X, Sezgin E, Hendrickson SL, Troyer JL, et al. The principal genetic determinants for nasopharyngeal carcinoma in China involve the HLA class I antigen recognition groove. PLoS Genet 2012;8(11):e1003103.

9 Yuan J, Liu S, Yu Q, Lin Y, Bi Y, Wang Y, et al. Down-regulation of human leukocyte antigen class I (HLA-I) is associated with poor prognosis in patients with clear cell renal cell carcinoma. Acta Histochem 2013;115(5):470-4.

10 Minami T, Minami T, Shimizu N, Yamamoto Y, De Velasco M, Nozawa M, et al. Identification of erythropoietin receptor-derived peptides having the potential to induce cancer-reactive 
cytotoxic T lymphocytes from HLA-A24(+) patients with renal cell carcinoma. Int Immunopharmacol 2014;20(1):59-65.

11 Tertipis N, Villabona L, Nordfors C, Nasman A, Ramqvist T, Vlastos A, et al. HLA-A*02 in relation to outcome in human papillomavirus positive tonsillar and base of tongue cancer. Anticancer Res 2014; 34(5):2369-75. Simons MJ, Chan SH, Wee GB, Shanmugarathnam K, Goh EH, Ho JH, et al. Nasopharyngeal carcinoma and histocompatibility antigens. IARC Sci Publ 1978;20:271-382.

Hildesheim A, Apple RJ, Chen CJ, Wang SS, Cheng Y-J, et al. Association of HLA class I and II alleles and extended haplotypes with nasopharyngeal carcinoma in Taiwan. J Natl Cancer Inst 2002;94:1780-9.

Hu SP, Day NE, Li DR, Luben RN, Cai KL, Ou-Yang T, et al. Further evidence for an HLA related recessive mutation in nasopharyngeal carcinoma among the Chinese. $\mathrm{Br} \mathrm{J}$ Cancer 2005;92(5):967-70.

15 Lu CC, Chen JC, Jin YT, Yang BH, Chan SH, Tsai ST. Genetic susceptibility to nasopharyngeal carcinoma within the HLA-A locus in Taiwanese. Int J Cancer 2003;103(6):745-51.

16 Sengupta S, den Boon JA, Chen IH, Newton MA, Dahl DB, Chen M, et al. Genome-wide expression profiling reveals EBV-associated inhibition of MHC class I expression in nasopharyngeal carcinoma. Cancer Res 2006;66(16):7999-8006.

17 Han L, Lin SJ, Pan JJ, Chen CB, Zhang Y, Zhang XC, et al. Prognostic factors of 305 nasopharyngeal carcinoma patients treated with intensity-modulated radiotherapy. Chin J Cancer 2010;29(2):145-50.

(Received May 18, 2016) 
Table 1 Univariate analysis of the prognostic role of various factors in the 117 NPC patients

\begin{tabular}{|c|c|c|c|c|c|c|c|c|c|c|c|c|c|c|}
\hline Variables & Item & $n$ & $\begin{array}{l}\text { LRFS } \\
(\%)\end{array}$ & $\chi^{2}$ & $P$ & $\begin{array}{c}\text { DMFS } \\
(\%)\end{array}$ & $\chi^{2}$ & $P$ & $\begin{array}{l}\text { DFS } \\
(\%)\end{array}$ & $\chi^{2}$ & $P$ & OS $(\%)$ & $\chi^{2}$ & $P$ \\
\hline \multirow{2}{*}{ Age } & $\leqq 50$ & 72 & 92.4 & 6.451 & 0.011 & 84.2 & 0.032 & 0.857 & 76.1 & 5.020 & 0.025 & 87.6 & 7.465 & 0.006 \\
\hline & $>50$ & 45 & 72.1 & & & 83.6 & & & 58.6 & & & 61.5 & & \\
\hline \multirow{3}{*}{ Sex } & Male & 81 & 84.2 & 0.006 & 0.938 & 83.5 & 0.870 & 0.351 & 68.0 & & & 73.8 & 0.357 & 0.550 \\
\hline & & & & & & & & & & 0.306 & 0.580 & & & \\
\hline & Female & 36 & 85.8 & & & 85.9 & & & 72.9 & & & 83.1 & & \\
\hline \multirow[b]{2}{*}{ Smoking history } & Yes & 54 & 80.5 & 0.317 & 0.573 & 83.2 & 0.432 & 0.511 & 63.4 & 1.529 & 0.216 & 66.0 & 2.998 & 0.083 \\
\hline & No & 63 & 91.6 & & & 84.3 & & & 77.7 & & & 93.1 & & \\
\hline \multirow{3}{*}{ Pathological type } & Non-keratinizing & 43 & 81.3 & & & 86.0 & 0.502 & 0.973 & 74.1 & 2.358 & 0.670 & 84.7 & 3.888 & 0.421 \\
\hline & & & & 7.120 & 0.130 & & & & & & & & & \\
\hline & Undifferentiated & 69 & 88.9 & & & 80.0 & & & 60.9 & & & 83.6 & & \\
\hline \multirow{6}{*}{ T stage } & & & & 3.260 & 0.353 & & 7.904 & 0.048 & 100 & 11.554 & 0.009 & & 3.685 & 0.298 \\
\hline & $\mathrm{T} 1$ & 9 & 100.0 & & & 100.0 & & & & & & 100.0 & & \\
\hline & & & & & & & & & .0 & & & & & \\
\hline & $\mathrm{T} 2$ & 23 & 90.9 & & & 93.9 & & & 89.7 & & & 94.1 & & \\
\hline & $\mathrm{T} 3$ & 44 & 86.8 & & & 85.8 & & & 79.0 & & & 76.0 & & \\
\hline & $\mathrm{T} 4$ & 41 & 76.8 & & & 69.3 & & & 41.2 & & & 68.3 & & \\
\hline \multirow{2}{*}{$\mathrm{N}$ stage } & No & 15 & 92.9 & 9.452 & 0.024 & 91.7 & 11.105 & 0.011 & 90.3 & 12.843 & 0.005 & 96.3 & 10.202 & 0.017 \\
\hline & N1 & 40 & 86.3 & & & 87.8 & & & 82.9 & & & 88.4 & & \\
\hline
\end{tabular}




\begin{tabular}{|c|c|c|c|c|c|c|c|c|c|c|c|c|c|c|}
\hline & $\mathrm{N} 2$ & 51 & 79.8 & & & 74.7 & & & 71.2 & & & 79.7 & & \\
\hline & N3 & 11 & 76.6 & & & 66.3 & & & 61.0 & & & 68.5 & & \\
\hline \multirow{4}{*}{ Clinical stage } & I & 2 & 100.0 & 2.805 & 0.423 & 100.0 & 5.119 & 0.163 & 100.0 & 8.472 & 0.037 & 100.0 & 2.928 & 0.403 \\
\hline & II & 15 & 92.9 & & & 100.0 & & & 0.917 & & & 91.7 & & \\
\hline & III & 50 & 88.4 & & & 89.2 & & & 0.809 & & & 79.0 & & \\
\hline & IV & 50 & 78.3 & & & 72.8 & & & 0.482 & & & 70.2 & & \\
\hline \multirow{2}{*}{ HLA-A $* 24$} & Positive & 50 & 0.749 & 0.635 & 0.426 & 0.799 & 0.620 & 0.430 & 0.749 & 0.635 & 0.426 & 0.645 & 2.932 & 0.087 \\
\hline & Negative & 67 & 0.868 & & & 0.872 & & & 0.868 & & & 0.822 & & \\
\hline \multirow{2}{*}{ HLA-B*46 } & Positive & 36 & 0.860 & 0.241 & 0.624 & 0.716 & 3.004 & 0.083 & 0.681 & 0.157 & 0.692 & 0.787 & 1.291 & 0.256 \\
\hline & Negative & 81 & 0.823 & & & 0.897 & & & 0.699 & & & 0.830 & & \\
\hline \multirow{2}{*}{ HLA-A*2-B*46 } & Positive & 31 & 90.0 & 2.810 & 0.094 & 0.664 & 8,362 & 0.003 & 0.635 & 0.014 & 0.907 & 0.798 & 0.730 & 0.393 \\
\hline & Negative & 86 & 79.9 & & & 0.903 & & & 0.715 & & & 0.845 & & \\
\hline
\end{tabular}

OS, overall survival; LRFS, local recurrence-free survival; DMFS, distant metastasis-free survival; DFS, disease-free survival 
Table 2 Cox multivariate analysis of the effect of multiple factors on nasopharyngeal carcinoma prognosis in the 117 patients

\begin{tabular}{|c|c|c|c|c|c|}
\hline Factors & $\beta$ & $S E$ & $\chi^{2}$ & $P$ & $R R(95 \% \mathrm{CI})$ \\
\hline \multicolumn{6}{|l|}{ LRFS } \\
\hline Age & 0.761 & 0.463 & 4.613 & 0.032 & $2.039(1.112-10.094)$ \\
\hline \multicolumn{6}{|l|}{ DMFS } \\
\hline HLA-A*02-B*46 & 1.016 & 0.206 & 5.027 & 0.024 & $2.762(1.024-7.450)$ \\
\hline $\mathrm{N}$ stage & 0.563 & 0.356 & 4.258 & 0.039 & $1.756(0.942-9.540)$ \\
\hline \multicolumn{6}{|l|}{ DFS } \\
\hline Age & 0.363 & 0.321 & 4.231 & 0.040 & $1.438(0.789-5.449)$ \\
\hline T stage & 0.520 & 0.219 & 5.734 & 0.017 & $1.682(0.799-7.577)$ \\
\hline $\mathrm{N}$ stage & 0.558 & 0.318 & 5.943 & 0.014 & $1.800(0.989-7.096)$ \\
\hline Clinical stage & 0.751 & 0.211 & 5.362 & 0.021 & $2.119(1.012-8.936)$ \\
\hline \multicolumn{6}{|l|}{ OS } \\
\hline Age & 0.588 & 0.393 & 6.210 & 0.013 & $1.800(0.799-8.232)$ \\
\hline $\mathrm{N}$ stage & 0.786 & 0.202 & 5.634 & 0.018 & $2.195(0.896-7.138)$ \\
\hline
\end{tabular}

OS, overall survival; LRFS, local recurrence-free survival; DMFS, distant metastasis-free survival; DFS, disease-free survival; CI, confidence interval 
Fig. 1 Role of HLA-A*02-B*46 haploid type in the prognosis of nasopharyngeal carcinoma patients without distant metastasis Comparison of total (A) and clinical stage III-IV (B) HLA-A*02-B*46 haplotype carriers $(n=31$ and 26 , respectively) and non-carriers ( $n=86$ and 74 respectively)

A

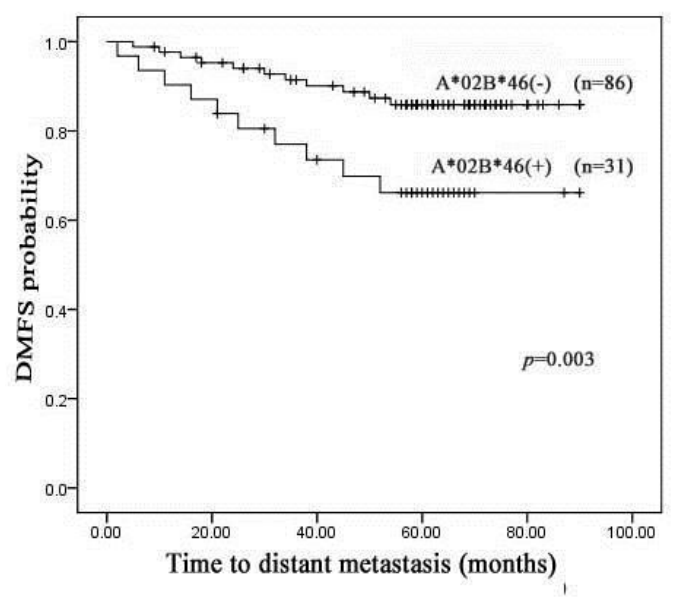

B

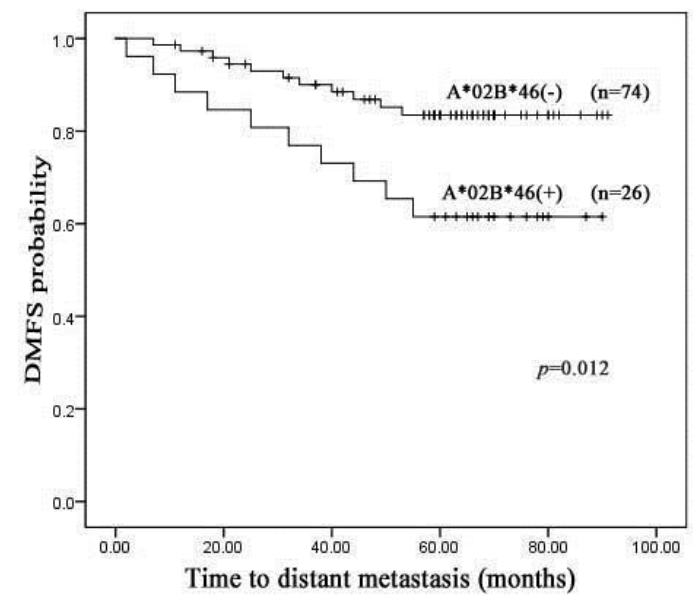

